\title{
Efectos de la interacción verbal en el desempeño de tareas cooperativas
}

\section{Verbal Interaction Effects on the Performance of Cooperative Tasks}

\author{
Nora Rangel \& Adriana Peña-Pérez Negrón \\ Universidad de Guadalajara, Guadalajara, México
}

\begin{abstract}
Resumen: En este trabajo se evaluó el efecto de permitir o restringir la interacción verbal entre díadas de participantes al realizar tareas cooperativas. Se analizó si el desempeño en la resolución de las tareas se vio afectado por las interacciones verbales de los participantes. La situación experimental (tarea) consistió en el armado de figuras tridimensionales en dos computadoras conectadas a través de internet. Para cada uno de los dos grupos experimentales, fueron asignados al azar 16 estudiantes universitarios. Un grupo tuvo que resolver la tarea sin la posibilidad de comunicación verbal, mientras que el otro grupo no tuvo tal restricción. Se observó que en las condiciones con posibilidad de interacción verbal, los participantes fueron más eficientes que en las condiciones en las que no se les permitió comunicarse. En el grupo con posibilidad de interacción verbal, se encontró que la mayoría de los participantes realizó comentarios directivos, aunque al parecer esto no afectó la eficiencia en el desempeño. En este trabajo se discuten los hallazgos en términos del tipo de respuesta requerido en la tarea, del papel del lenguaje en las interacciones y de las relaciones establecidas entre los participantes involucrados, los que podrían facilitar la ejecución de tareas cooperativas.
\end{abstract}

Palabras clave: cooperación, contingencia compartida, respuestas individuales, respuestas compartidas, interacción verbal.
Abstract: This study was conducted to evaluate the effect of allowing or restricting verbal interaction between dyads of participants when performing cooperative tasks. It was also analyzed whether the performance when solving the task was affected by the participants' verbal interactions. The experimental setting entailed the assembly of tridimensional figures in two computers connected through internet. Sixteen undergraduate students were randomly assigned to each experimental group. One group had to solve the task without the aid of verbal communication, while the other did not have this restriction. It was observed that the participants, which were allowed to interact were more efficient than the ones who were not allowed to communicate with each other. Most of the participants who were able to interact verbally made directive statements, but apparently this did not affect the performance's efficiency. Results are discussed in terms of the type of answer required for the task, the language role in the interactions and the established relationships between the participants involved, which may ease the execution of cooperative tasks.

Keywords: cooperation, shared contingency, individual responses, shared responses, verbal interaction.

Contacto: N. Rangel. Centro de Estudios e Investigaciones en Comportamiento, Francisco de Quevedo \#180; Col. Arcos Vallarta; Guadalajara, Jal., México; C.P. 44130. Correo electrónico: nora.rangel@ academicos.udg.mx

Cómo citar: Rangel, N. \& Peña-Pérez Negrón, A. (2016). Efectos de la interacción verbal en el desempeño de tareas cooperativas. Revista de Psicología, 25(1), 1-19.

http://dx.doi.org/10.5354/0719-0581.2016.40628 


\section{Introducción}

En la definición del comportamiento social, desde una perspectiva psicológica, tradicionalmente se ha asumido que la presencia de dos o más organismos en una situación es el criterio definitorio para que emerja la naturaleza social de la interacción entre ellos, o de ambos, respecto de un mismo ambiente. En la mayoría de los casos, esta definición ha sido compartida por etólogos, debido a que parece ser capaz de describir el comportamiento en conjunto de organismos de cualquier especie (e.g., Etkin, 1964; Pierce, 1991; Secord \& Backman, 1974; Skinner, 1953). Sin embargo, Ribes-Iñesta, Rangel y LópezValadéz (2008) han afirmado que cuando se asume esta concepción, no se toma en cuenta la estructura funcional del ambiente en el que la interacción se presenta y, en consecuencia, "el medio o ambiente social no precede a la interacción social, sino que es un resultado de ella" (p. 45).

Desde una perspectiva interconductual, específicamente desde la propuesta realizada por Ribes-Iñesta (2001) y RibesIñesta, Rangel y López-Valadéz (2008), el comportamiento social está determinado por la naturaleza del medio que posibilita este tipo de interacciones, por lo que lo social no puede, en definitiva, ser un efecto de la interacción misma que se da entre los organismos. Se asume, de este modo, que el medio de contacto que posibilita el comportamiento social es convencional y, en tanto, que dicho medio es articulado y mediado por el lenguaje, es exclusivo de los humanos.

Basado en lo anterior, no es de extrañar que -a lo largo de varias décadas de investigación experimental acerca del comportamiento social- se haya observado que el establecimiento de comunicación verbal entre los participantes de una inter- acción, haya favorecido tanto la preferencia como el desempeño de tareas cooperativas ${ }^{1}$ (Bicchieri \& Lev-On, 2007; Cohen, Wildschut, \& Insko, 2010; Hake \& Schmid, 1981; Marwell \& Schmitt, 1975; Meleady, Hopthrow, \& Crisp, 2013; Pulido, Rangel, \& Ortiz, 2013; Pulido-Avalos, RangelBernal, \& Ortiz-Rueda, 2014; Ribes Iñesta, Rangel, Magaña, López, \& Zaragoza, 2005; Wichman, 1970).

Tampoco sorprende que, en consecuencia, la interacción verbal se haya considerado una de las principales variables que afectan el comportamiento cooperativo cuando se utilizan procedimientos que involucran dilemas sociales (e.g., Bicchieri \& LevOn, 2007; Dawes \& Messick, 2000; Sally, 1995). En estos procedimientos, los participantes son expuestos a dos alternativas, una calificada como no cooperativa y la otra como cooperativa. La elección de la primera alternativa significa el beneficio individual máximo; mientras que elegir la segunda, significa reducir el beneficio individual con el propósito de obtener un beneficio mayor para el colectivo (Balliet, Mulder, \& Van Lange, 2011; Marwell \& Schmitt, 1975). De acuerdo con Bicchieri y Lev-On (2007), utilizando dichos procedimientos se han observado niveles mínimos de cooperación cuando los participantes no han interactuado verbalmente durante la realización de la tarea, en comparación a condiciones en las que sí se comunican entre ellos.

\footnotetext{
${ }^{1}$ En el presente trabajo se suscribe el tratamiento que hacen de la cooperación Ribes-Iñesta et al. (2006, 2010) y Pulido Avalos, Rangel Bernal, Ávila Hernández, Mérida Velez y Ribes Iñesta (2014). En ese sentido, a lo largo este documento se hace referencia solo a situaciones cooperativas de altruismo parcial, en las que las respuestas de los individuos involucrados en una situación producen resultados positivos para todos los participantes que se encuentran resolviendo la tarea experimental. Debido a que en estas situaciones lo que hace uno de los participantes afecta a otro(s), se habla de contingencias compartidas (ver Ribes, Rangel, \& López, 2008).
} 
Esta preferencia por el comportamiento cooperativo, no solo se ha estudiado a través de dilemas sociales, sino que también se ha analizado a través de tareas de ejecución; como por ejemplo, tareas de igualación de la muestra o juegos de rompecabezas virtuales o reales. El comportamiento cooperativo se ha estudiado, principalmente, desde dos tradiciones importantes dentro de la psicología experimental: la perspectiva operante y la perspectiva interconductual (e.g., Azrin \& Lindsley, 1956; Hake \& Vukelich, 1972; Marwell \& Schmitt, 1975; Pulido-Avalos et al., 2014; Ribes Iñesta et al., 2005; RibesIñesta et al. 2006; Schmid \& Hake, 1983; Schmitt, 1984, 1986, 1987, 1998, 2000).

Aunque desde la perspectiva operante se ha reportado el efecto favorecedor de la interacción verbal, que se establece entre los participantes sobre la preferencia del comportamiento cooperativo (e.g., Hake \& Schmid, 1981; Marwell \& Schmitt, 1975), al parecer, esta variable no ha sido explorada sistemáticamente. El estudio de este tipo de comportamiento se ha centrado en la variación del tipo de respuestas y de la cantidad de respuestas y/o reforzadores obtenidos por la elección de respuestas cooperativas y no cooperativas (e.g., Hake, Olvera, \& Bell, 1975; Matthews, 1977; Shimoff \& Matthews, 1975; Spiga, Cherek, Grabowski, \& Bennet, 1992).

Por otra parte, es desde la perspectiva interconductual y, específicamente, desde la propuesta de análisis de las dimensiones funcionales del comportamiento social (cf. Ribes-Iñesta, 2001; Ribes-Iñesta, Rangel, \& López-Valadéz, 2008) que se ha iniciado una exploración sistemática de los efectos de las interacciones verbales sobre la elección de contingencias compartidas. Es decir, contingencias que afectan a todos los individuos en la interacción, en las que la cooperación entre los participantes es requerida, en contraste con contingencias individuales que solo afectan al individuo que responde (cf. Pulido Avalos, 2012; Pulido-Avalos et al., 2014; Ribes Iñesta et al., 2005; RibesIñesta et al., 2006; Ribes-Iñesta, Rangel, Ramírez, et al., 2008). Como un efecto sistemático en los estudios realizados bajo esta propuesta, se ha encontrado que el establecimiento de intercambios verbales entre los participantes expuestos a la situación experimental, ha afectado positivamente la elección de contingencias compartidas.

Sin embargo, Pulido et al. (2013) han señalado que en el estudio de la preferencia por contingencias individuales versus compartidas, ambas alternativas se han presentado de manera concurrente en la situación experimental y consideraron que la presentación de cada una de ellas por separado, permitiría evaluar su efecto de manera independiente en el comportamiento de los participantes. Por ello, estos autores realizaron un estudio en el que se eliminó dicha concurrencia y expusieron a los participantes a la resolución de tareas bajo contingencias individuales, colectivas o compartidas con el objetivo de explorar su efecto de manera aislada, sobre el establecimiento y el tipo de intercambios verbales entre los participantes y sobre su desempeño instrumental.

Como procedimiento de control, Pulido et al. (2013) restringieron en algunas díadas la posibilidad de comunicación. Observaron que cuando los participantes fueron expuestos a las distintas contingencias y les fue permitido comunicarse entre ellos, mostraron mejores ejecuciones que en condiciones de imposibilidad lingüística (los participantes que se comunicaron entre ellos tardaron menos tiempo y cometieron menos errores en la resolución de la tarea experimental), sobre todo en la con- 
tingencia compartida y, que dependiendo del tipo de contingencia a la que fueron expuestos, los participantes mostraron intercambios verbales de distintas cualidades, es decir, referidos a distintas características de la situación experimental.

Es importante mencionar que en el estudio de Pulido et al. (2013), durante la contingencia compartida, se necesitó de la acción de los dos participantes de la díada para ejecutar una misma respuesta (respuestas compartidas). Al respecto, los autores del presente trabajo consideraron que fue quizá este requisito el que "obligó" a los participantes a interactuar verbalmente (debido a la coacción requerida por parte de los dos participantes, para la realización de cada respuesta).

Dados estos antecedentes, parecería plausible preguntarse si el establecimiento de interacción verbal entre los participantes afectaría las medidas de desempeño instrumental de tareas cooperativas, particularmente, en tareas en las que, a diferencia del procedimiento de Pulido et al. (2013) se requirieran respuestas individuales; es decir, respuestas realizadas por cada participante, de manera independiente, con el objetivo de realizar una tarea común. Así, el objetivo principal del presente experimento fue evaluar el efecto de permitir o restringir la comunicación entre los participantes sobre el desempeño en una tarea cooperativa que requirió respuestas individuales.

Al mismo tiempo, considerando lo reportado por Pulido et al. (2013) respecto de la influencia del tipo de contingencia sobre la cualidad de los intercambios verbales, y tomando en cuenta que las contingencias a las que fueron expuestos los participantes del presente estudio fueron compartidas, se analizó si durante las condiciones de posibilidad de comunicación, se presentaron interacciones verbales por parte de alguno de los participantes que plantearan estrategias de resolución y, de ser así, si este tipo de interacciones afectaron el desempeño instrumental de la tarea.

En este mismo sentido, en la literatura se ha reportado repetidamente que en situaciones cooperativas, las interacciones verbales que se establecen entre los participantes van dirigidas a proponer estrategias de resolución de la tarea. Esto se ha relacionado con la emergencia de liderazgo en la situación experimental (e.g., Bicchieri \& Lev-On, 2007; Bruttel \& Fischbacher, 2013; Sutter $\&$ Rivas, 2014), aspecto que se sugiere podría estar influyendo en un mejor desempeño por parte de los participantes.

A su vez, desde una perspectiva interconductual, Rangel y Ribes (2009) y RibesIñesta, Rangel y López Valadéz (2008) han reconocido que el liderazgo es una de las posibles relaciones que pueden establecerse en interacciones diádicas (como unidad mínima de análisis). Estos autores plantean que el liderazgo se puede identificar cuando uno de los individuos sigue las directrices de otro, sin necesidad de estar bajo una relación de poder-autoridad formalmente instituida. Dicho aspecto se cumple entre los participantes de este tipo de preparaciones experimentales, y bajo la suposición de que el o los individuos que siguen dichas directrices reconocen en el otro (el llamado líder), una autoridad moral - a diferencia de la autoridad formal- lo que le da el derecho de guiar su comportamiento ${ }^{2}$.

\footnotetext{
${ }^{2}$ La autoridad moral se refiere al reconocimiento que uno o varios individuos otorgan a otro, con base en su prestigio, conocimiento, habilidad o carisma, lo que le da un derecho -desde el punto de vista de los primeros- a guiar su comportamiento en una situación determinada. La autoridad formal se refiere al reconocimiento de que un individuo tiene el derecho de mandar a otro(s), con base en relaciones de poder dadas en estructuras jerárquicas formalmente instituidas (ver Rangel \& Ribes, 2009; Ribes-Iñesta, Rangel y López Valadéz, 2008).
} 
Con base en lo anterior, un objetivo adicional del presente estudio fue analizar si bajo una situación cooperativa que requirió respuestas individuales, emergía un líder entre los participantes involucrados en la realización de la tarea, evaluando su efecto en el desempeño de la misma.

\section{Método}

\section{Participantes}

Participaron en el estudio 16 estudiantes universitarios (13 hombres y 3 mujeres), cuyas edades oscilaron entre los 18 y los 19 años de edad, quienes colaboraron voluntariamente en el experimento a cambio de objetos de escritorio (i.e., bolígrafos, lapiceros, marcadores de texto, cuadernos) que pudieron elegir al finalizar el estudio.

\section{Aparatos, materiales y situación expe- rimental}

Las sesiones tuvieron lugar en un salón iluminado y libre de distractores, con dos escritorios que se daban la espalda y separados por un librero alto. En cada escritorio se encontraba una computadora con monitor LCD Alienware modelo OptX AW2310 de 23" a 120Hz, teclado y ratón. En cada sesión ambas computadoras estuvieron conectadas mediante internet y se registraron automáticamente las respuestas de los participantes.

Se utilizaron también audífonos con micrófonos, para facilitar o interferir la interacción verbal entre los participantes, dependiendo de la condición experimental, y un software que consistió en un entorno virtual colaborativo tridimensional, en el que cada uno de los usuarios pudo ver el mismo escenario virtual desde su propia perspectiva, la que correspondía al lugar en el que se ubicaba dentro del mundo virtual.

Las sesiones fueron grabadas mediante la aplicación aTube Catcher (versión 3.8; Uscanga, 2011) que también registró, el intercambio verbal entre los participantes. Por otra parte, se desarrolló un software ad hoc para el cálculo de los índices de ineficiencia en el desempeño de la tarea.

\section{Diseño}

Se utilizó un diseño de replicación intra y entre participantes que permitió, a su vez, la comparación entre grupos. Los participantes fueron asignados al azar a uno de los dos grupos experimentales que difirieron en la secuencia en la que fueron expuestos a las condiciones experimentales.

Todas las díadas fueron expuestas a una sesión de demostración de la tarea, en la que se instruyó a los participantes en el manejo del software. Posteriormente, ambos grupos fueron expuestos a dos fases experimentales, de tres sesiones cada una. Durante la primera fase, las díadas del Grupo 1 resolvieron la tarea con la posibilidad de interactuar verbalmente entre ellos, mientras que en la segunda fase, no se les permitió interactuar verbalmente.

En el Grupo 2 se invirtió el orden de las condiciones, durante la primera fase no se permitió la interacción verbal entre los participantes de las díadas, mientras que en la segunda fase experimental los participantes pudieron interactuar verbalmente entre ellos. La tabla 1 presenta el diseño experimental utilizado. 
Tabla 1

Diseño experimental

\begin{tabular}{lccc}
\hline & \multicolumn{3}{c}{ Fases } \\
\cline { 2 - 4 } & 1 (una sesión) & 2 (tres sesiones) & 3 (tres sesiones) \\
\hline Grupo 1 & Demostración de la & Con posibilidad de & Sin posibilidad de \\
$n=4$ díadas & tarea & interacción verbal & interacción verbal \\
Grupo 2 & Demostración de la & Sin posibilidad de & Con posibilidad de \\
$n=4$ díadas & tarea & interacción verbal & interacción verbal \\
\hline
\end{tabular}

\section{Procedimiento}

El entorno virtual colaborativo consistió en una "isla" en la que se pusieron desordenadamente 14 piezas; formadas, cada una de ellas, con distintas cantidades de cubos (ver tabla 2) de diferentes colores, que los participantes, agrupados en díadas, tenían que utilizar para formar una figura geométrica (ver figura 1).
Los participantes tuvieron que armar seis figuras distintas, una para cada sesión experimental, con el objetivo de evitar un efecto de aprendizaje (ver tabla 3). Al inicio de cada sesión experimental, se mostró a los participantes la figura que tenían que armar en un modelo hecho con piezas de plástico (ver figura 2). Dicha figura permaneció a la vista de los participantes durante toda la sesión experimental.

\section{Tabla 2}

Piezas para armar el cubo en cada sesión

\begin{tabular}{cccc}
\hline Cantidad de piezas & Cubos unidos & Forma & Ejemplo visual \\
\hline 2 & 1 & Cubo & \\
5 & 2 & Rectángulo & \\
5 & 3 & Rectángulo & \\
2 & 3 & Ángulo & \\
\hline
\end{tabular}
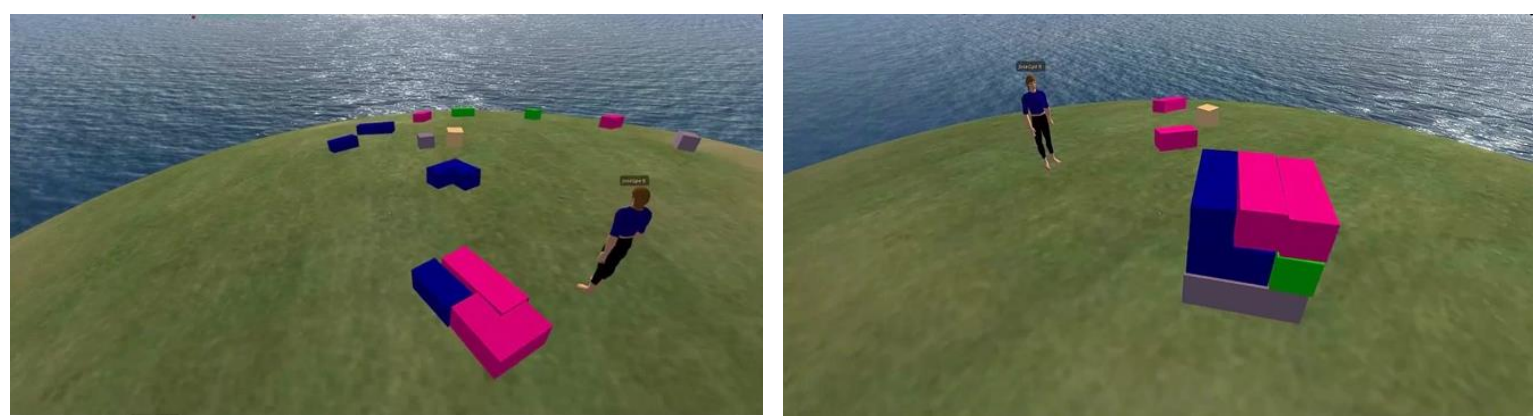

Figura 1. Escenario del entorno virtual colaborativo para la realización de la tarea, así como un ejemplo de una figura terminada. 
Tabla 3

Figuras a armar en cada sesión

\begin{tabular}{|c|c|}
\hline Sesión & Figura \\
\hline 1 & Ortoedro $4 \times 3 \times 2$ \\
\hline 2 & Cubo $3 \times 3 \times 3$ \\
\hline 3 & Ortoedro $4 \times 2 \times 3$ \\
\hline 4 & $\begin{array}{l}\text { Cruz con brazos } \\
\text { de } 8 \text { cubos }\end{array}$ \\
\hline 5 & $\begin{array}{l}\text { Cubo } 3 \times 3 \times 3 \text { sin } \\
\text { el cubo del centro }\end{array}$ \\
\hline 6 & $\begin{array}{l}\text { Escalera de } 3 \text { de } \\
\text { ancho y con } 4 \\
\text { escalones }\end{array}$ \\
\hline
\end{tabular}

En la aplicación, cada usuario estuvo representado gráficamente mediante un avatar humanoide. Sin embargo, los sujetos no podían verse a sí mismos en el mundo virtual, lo que significa que los avatares funcionaron en primera persona, tal y como sucede en el mundo real. El avatar correspondió al género del usuario y tenía su nombre escrito en la pantalla para facilitar la identificación y comunicación, cuando lo permitió el diseño experimental (ver figura 1).

Los usuarios pudieron interactuar con el escenario, mediante combinaciones de teclas y el uso del ratón, realizando las siguientes tareas: navegación (caminar o volar sobre la isla) y selección de objetos, su movimiento y su rotación. Durante todas las sesiones, los participantes contaron con una hoja, detallando las combinaciones de teclas, y un ratón para interactuar con el entorno y con sus com- pañeros ${ }^{3}$. No hubo tiempo límite para resolver la tarea.

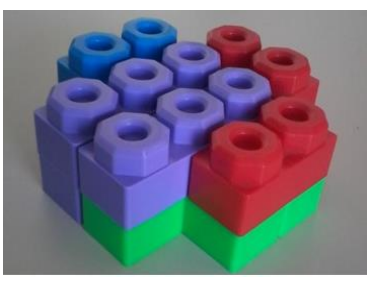

Figura 2. Ejemplo de figura hecho con piezas de plástico mostrado a los participantes durante la sesión.

Antes de iniciar el experimento, se mostró a los participantes la manera de interactuar con los objetos del escenario, la forma de navegación y, según fuera el caso, la forma de comunicación entre ellos. Posteriormente, se les permitió familiarizarse con la aplicación por un lapso de 2 a 3 minutos, antes de presentárseles verbalmente las siguientes instrucciones:

Van a armar entre los dos una figura como esta con los cubos que se encuentran en el escenario [se les mostraba la figura, además de decirles de qué tipo era. Por ejemplo, un cubo de $3 \times 3$ o una cruz]. No todas las piezas son necesarias para armar la figura. Nosotros estamos aquí a un lado, cuando terminen, por favor, avísenos.

En caso de que la comunicación verbal estuviera permitida, se agregaba lo siguiente: "Para comunicarse, usen el micrófono que está integrado a los audífonos".

En caso de que no se les permitiera tener comunicación, se les indicaba lo siguiente: "Por favor, no se comuniquen entre ustedes".

\footnotetext{
${ }^{3}$ Para la navegación del avatar se utilizaron las flechas o teclas "WASD", para el movimiento de la cabeza se utilizó el ratón. La selección y el movimiento de los objetos se realizaron mediante el ratón, mientras que la rotación de los mismos se realizó mediante el ratón y las teclas "Ctrl + Shift".
} 


\section{Análisis de datos}

En coherencia con la propuesta teórica, y de acuerdo con la lógica del diseño utilizado en este trabajo, se realizaron análisis de datos individuales (cf. Acuña, 2010).

Con el objetivo de evaluar el desempeño instrumental de la tarea, se calcularon dos índices de ineficiencia en la ejecución de la tarea de cada una de las díadas, respecto de la distancia recorrida por los objetos en cada una de las sesiones experimentales: el Índice de Ineficiencia Final (IIneF) y el Índice de Ineficiencia Total (IIneT).

El IIneF se calculó respecto de los objetos que formaron parte de la figura final, mientras que el IIneT se calculó en relación con la totalidad de los objetos movidos -como se mencionó, no se requería de todos los objetos disponibles para formar la figura solicitada-. En ambos casos, se tomó como medida base la distancia recorrida para cada objeto desde su origen, hasta el lugar en el que terminaron al formar la figura. Este sería el recorrido más eficiente, dato que se utilizó como unidad.

Posteriormente, a la distancia que realmente recorrió el objeto se le restó esta unidad y se utilizó como divisor para obtener el IIneF. Por ejemplo, si la distancia ideal es de 5 y la distancia que recorrió el objeto fue de 32 , entonces $32-5=27 / 5=$ 5,4 ; esto es, los participantes recorrieron 5,4 veces más distancia que la necesaria para colocar un objeto en su lugar.

Para calcular el IIneT, se sumó la distancia implicada en estos movimientos al total anterior; es decir, si los participantes movieron un objeto 10 unidades y este no fue parte de la figura final, entonces, utilizando los datos anteriores, $27+10=$ 37/5; el IIneT = 7,4.
De acuerdo con las hipótesis que guiaron el presente trabajo, se esperaba que las díadas con posibilidad de interacción verbal mostraran menores índices de ineficiencia en comparación con aquellas a las que no se les permitió interactuar verbalmente durante la resolución de la tarea.

De acuerdo con Pulido et al. (2013) las díadas más eficientes no solo mostraron más precisión durante la realización de la tarea, sino que la resolvieron en un lapso de tiempo menor. Por lo anterior, se propone también como un indicador de eficiencia, el tiempo en el que las díadas resolvieron la tarea; esperando en el mismo sentido, que esta medida se vea afectada por la permisión o la restricción de la interacción verbal entre los participantes.

Finalmente, se presentó el número de interacciones verbales (totales y directivas o estratégicas) establecidas entre los participantes, así como los datos por participante, de la distancia recorrida por los objetos y del número de movimientos realizados a lo largo de las sesiones (a diferencia de los índices de ineficiencia en los que se presentó el dato por díada).

Lo anterior, con el objetivo de observar si los participantes con un mayor número de interacciones verbales $\mathrm{y}$, sobre todo, de intervenciones directivas y/o estratégicas, fueron los que se mostraron más eficientes, de manera individual, durante las sesiones experimentales; comparando su desempeño en sesiones en las que no se les permitió interactuar verbalmente entre ellos. Una interacción verbal fue registrada cada vez que un participante emitía algún comentario. Las intervenciones directivas o estratégicas se definieron como aquellas que, realizadas por uno de los participantes, sugerían o indicaban al otro el movimiento de alguna pieza o al- 
guna acción en particular para completar la figura solicitada.

\section{Resultados}

En la figura 3 se presenta una comparación entre el IIneF y el IIneT de cada díada en los dos grupos. Se puede observar que en el Grupo 1 las dos díadas que mostraron los índices más altos de ineficiencia (IIneF e IIneT) fueron la Díada 2 y la Díada 3 durante la Fase 2, en la que no se permitió comunicación entre los participantes. La Díada 2 en la primera sesión sin interacción verbal (SI1) alcanzó un IIneF de 32,19 y un IIneT de 36,52; mientras que la Díada 3 en la segunda sesión sin interacción verbal (SI2) alcanzó un IIneF de 21,34 y un IIneT de 25,05.

Aunque las Díadas 1 y 4 de este mismo grupo alcanzaron sus más altos índices de ineficiencia (IIneF e IIneT) durante la Fase 1, en la que se permitió que los participantes se comunicaran, estos índices fueron menores (casi por mitad) que el de las Díadas 2 y 3 en la Fase 2, expuestas a restricción verbal. La Díada 1 en SI1 alcanzó un IIneT de 16,22; mientras que la Díada 4 en SI2 obtuvo un IIneF de 13,69 y un IIneT de 13,96.

En lo que respecta al Grupo 2, tres de las díadas (la 6 en SI3, la tercera sesión sin interacción verbal, y las Díadas 7 y 8 en SI1) mostraron los mayores índices de ineficiencia (IIneF e IIneT) durante la Fase 1 en la que se restringió la comunicación entre los participantes.

Por su parte, la figura 4 muestra la duración de las sesiones (en minutos y segundos) para ambos grupos. En el Grupo 1, todas las díadas alcanzaron la mayor duración en la resolución de la tarea durante la primera sesión del experimento (con comunicación). En dos de las díadas (la 1 y la 4), el tiempo en el que se resolvió la tarea fue disminuyendo: a partir de la tercera sesión con comunicación verbal (I3), en la Fase 1, la Díada 4 tardó entre 3 y 4 minutos en terminar el armado de la figura; mientras la Díada 1, tomó alrededor de 5 minutos.

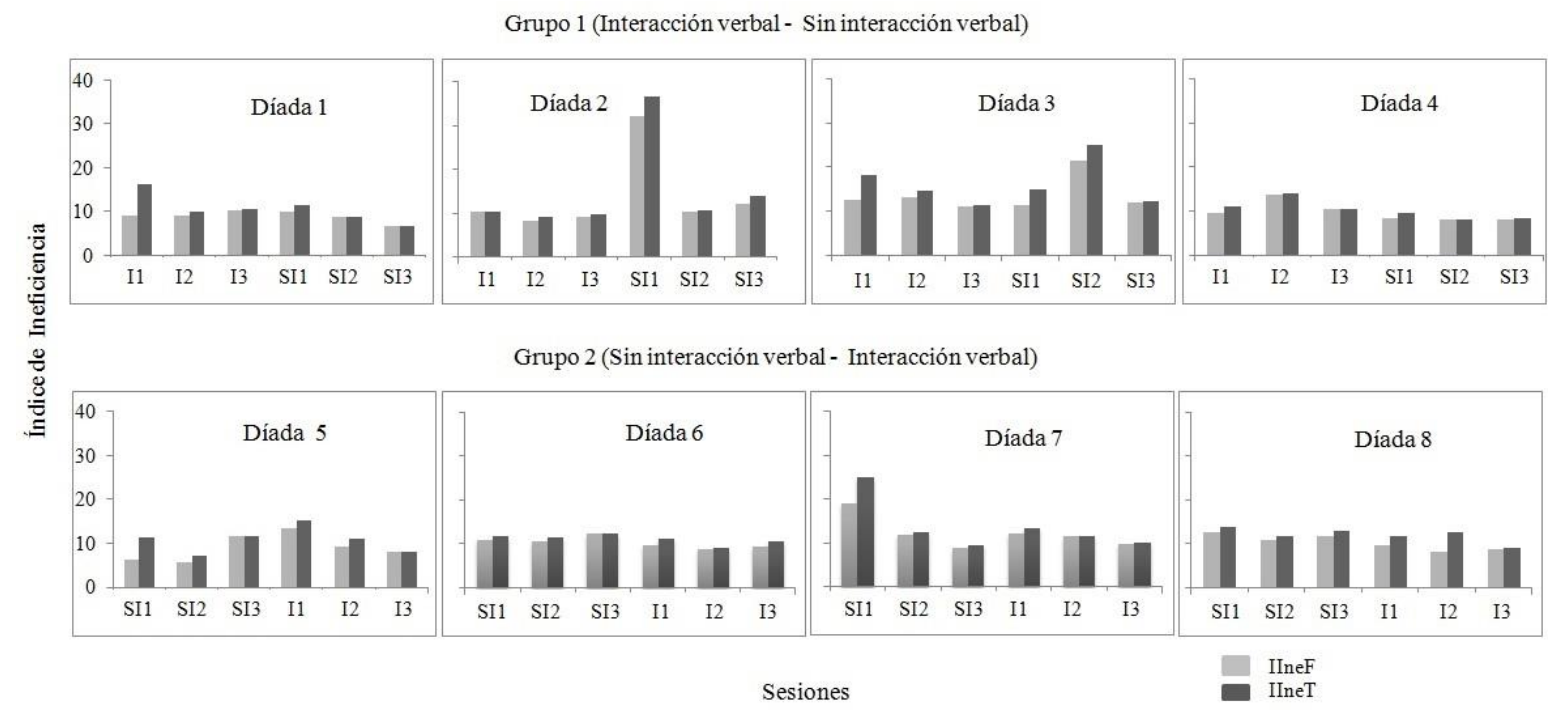

Figura 3. IIneF = Índices de Ineficiencia Final; IIneT = Índice de Ineficiencia Total; SI = Sesiones sin interacción verbal (el número corresponde al número de sesión); $\mathrm{I}=$ Sesiones con interacción verbal (el número corresponde al número de sesión). 


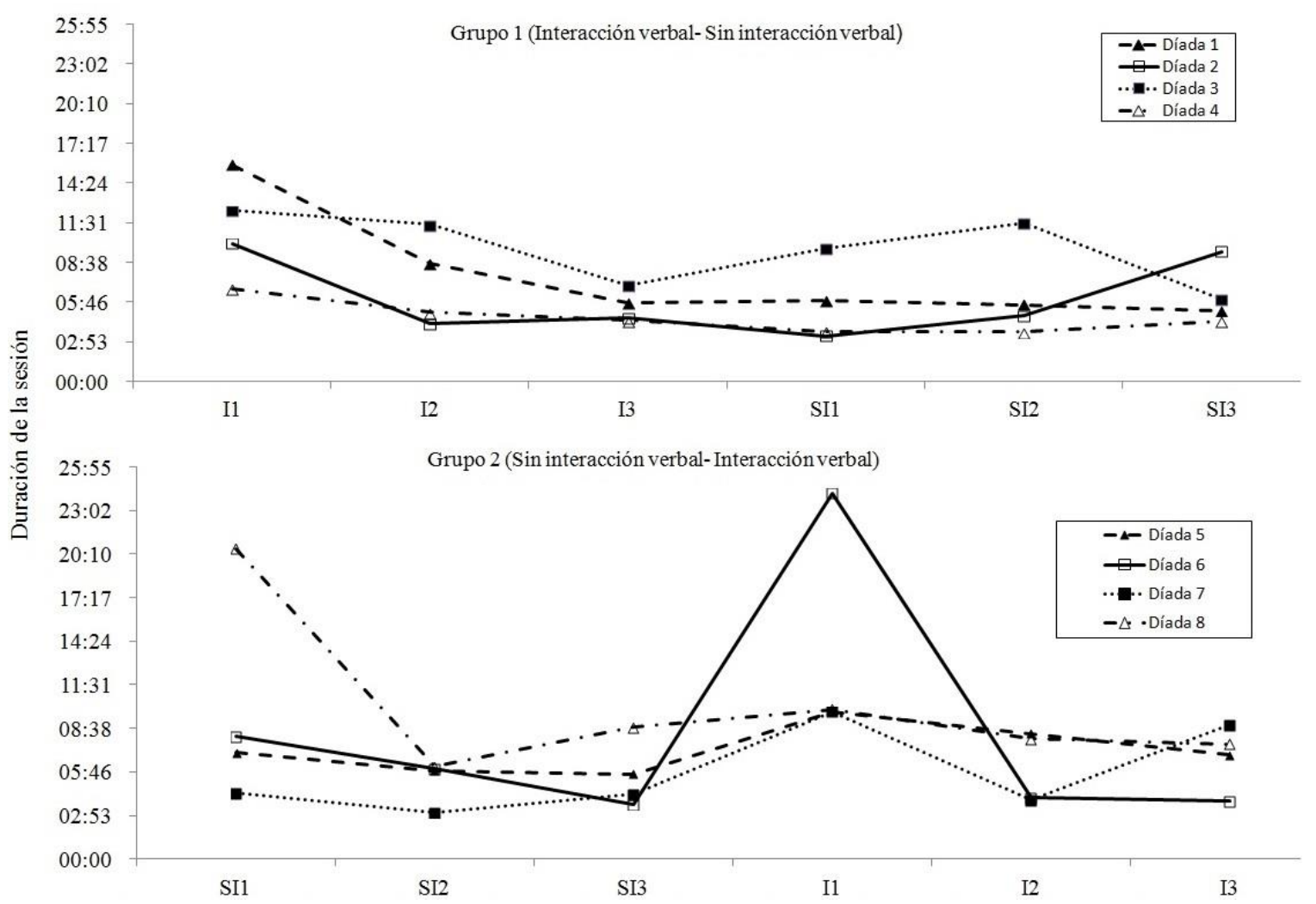

Figura 4. Minutos y segundos que tardaron las díadas en la resolución de la tarea. SI = Sesiones sin interacción verbal (el número corresponde al número de sesión); I = Sesiones con interacción verbal (el número corresponde al número de sesión).

Por otra parte, las Díadas 2 y 3 disminuyeron el tiempo de armado en las sesiones I2 e I3, respecto de la primera sesión con comunicación (I1) de la Fase 1. No obstante, el tiempo se volvió a incrementar en algunas sesiones de la Fase 2, llegando a niveles cercanos a la duración de SI1 (Díada 3 en SI1 y SI2, y Díada 2 en SI3).

En el Grupo 2 (con excepción de la Díada 8 , que alcanzó la mayor duración en la primera fase, en SI1 con 20 minutos y 31 segundos), el resto de las díadas lo hicieron durante I1 en la Fase 2, es decir, en la primera sesión en la que se permitió comunicación entre los participantes, incluso la Díada 8 mostró un incremento en el tiempo de armado de la figura solicitada durante esta misma sesión (I1).
En tanto, la figura 5 muestra el número de intervenciones verbales realizadas por cada uno de los participantes en la Fase 1, para el Grupo 1, y en la Fase 2, para el Grupo 2; fases en las que se permitió la comunicación entre los participantes, tal y como se señala en el diseño experimental utilizado.

Las barras en la figura muestran el total de intervenciones verbales realizadas por cada participante, mientras que la parte punteada en cada barra muestra el número de intervenciones que fueron directivas (aquellas que indicaban, sugerían o solicitaban al otro participante mover o no ciertas piezas durante la sesión experimental, o seguir ciertas estrategias en la resolución de la tarea). 
Únicamente se registraron las intervenciones directivas que fueron atendidas por el otro participante durante el desarrollo de la tarea experimental. El registro de estas interacciones fue realizado por dos observadores independientes. La confiabilidad del análisis se calculó dividiendo el número de acuerdos entre el número total de observaciones, en todas las sesiones el índice fue mayor al $80 \%$.

Con excepción de la Díada 2 (Grupo 1), cuyas intervenciones verbales fueron prácticamente nulas, todas las díadas se comunicaron entre ellas cuando tuvieron la oportunidad de hacerlo. En la mayoría de las sesiones se presentaron comentarios directivos o estratégicos por parte de los dos participantes (excepto en SI2 de la Díada 6 y en SI3 de la Díada 8, en la que uno de los participantes no realizó este tipo de comentarios).

En general, en dos díadas del Grupo 1 (Díadas 3 y 4) y en tres del Grupo 2 (Díadas 6, 7 y 8), se puede observar que uno de los participantes tuvo una mayor cantidad de intervenciones directivas respecto de su compañero de díada, estos fueron los participantes $6,7,11,14$ y 16 . En el resto de las díadas, el participante que tuvo una mayor cantidad de este tipo de intervenciones varió en las distintas sesiones en las que se les permitió comunicarse.

Grupo 1 (Interacción verbal- Sin interacción verbal)

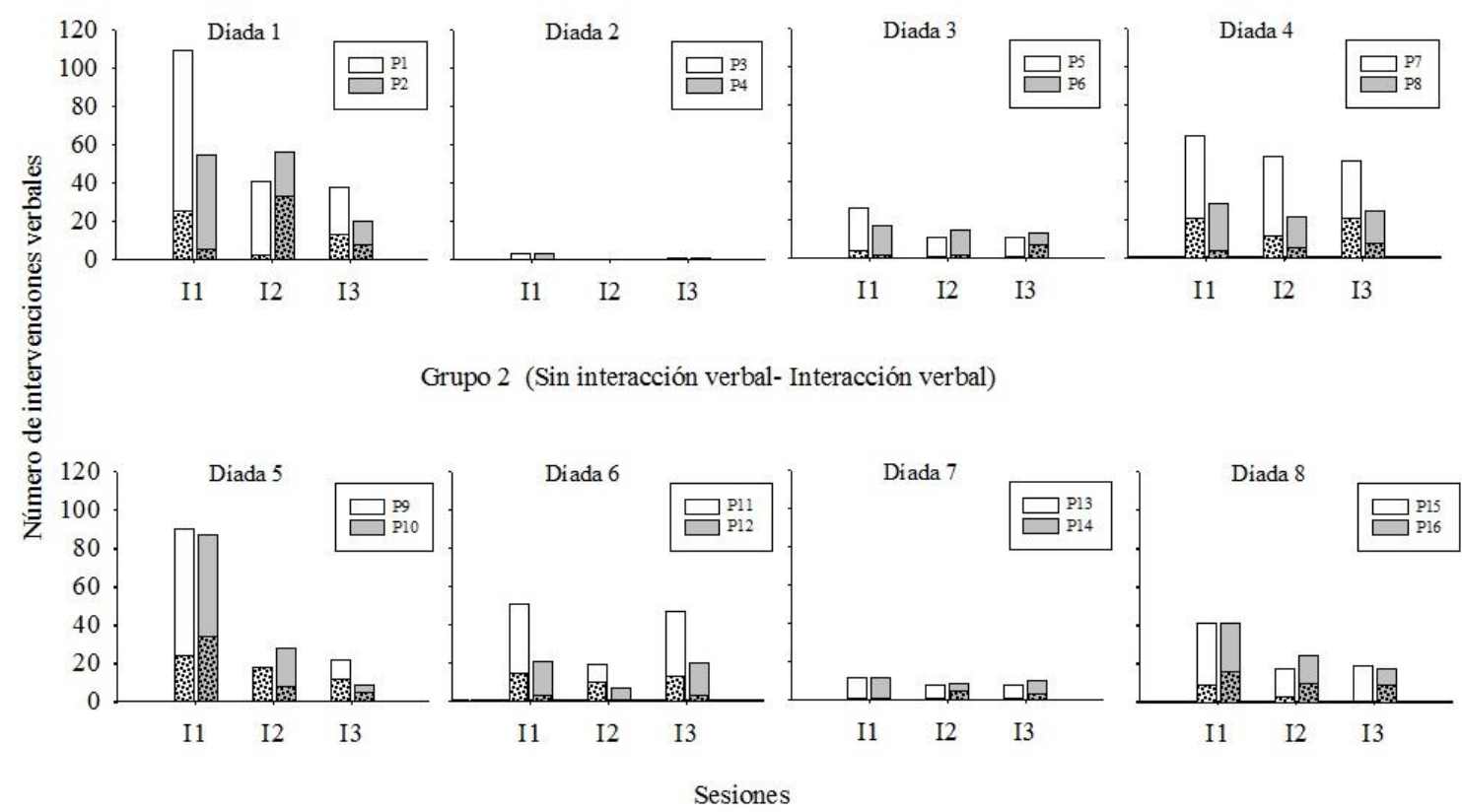

Figura 5. Número total de intervenciones verbales realizadas por cada uno de los participantes durante la condición de posibilidad de interacción verbal. Los participantes del Grupo 1 se expusieron a esta condición durante la Fase 1 y los participantes del Grupo 2 lo hicieron durante la Fase 2. La parte punteada de cada barra muestra el número de intervenciones que fueron directivas. $\mathrm{P}=$ Participantes; $\mathrm{SI}=$ Sesiones sin interacción verbal (el número corresponde al número de sesión); I = Sesiones con interacción verbal (el número corresponde al número de sesión). 
Por su parte, la figura 6 muestra los datos, por participante, de la distancia recorrida por los objetos. En general, se puede observar que en las díadas hubo un participante $(3,5,7,9,11,13$ y 15) que durante todas o la mayoría de las sesiones recorrió una mayor distancia con objetos que su compañero (exceptuando la Díada 1, en la que un participante recorrió más distancia en tres sesiones y el otro en las otras tres).
Por último, la figura 7 muestra los datos, por participante, del número de movimientos realizados a lo largo de las sesiones. Se puede observar también que en todas las díadas uno de ellos $(2,3,6,11,13$ y 15) movió más objetos que el otro en la mayoría de las sesiones (con excepción de las Díadas 4 y 5 , en las que un participante movió más objetos en tres sesiones y el otro participante en las otras tres).

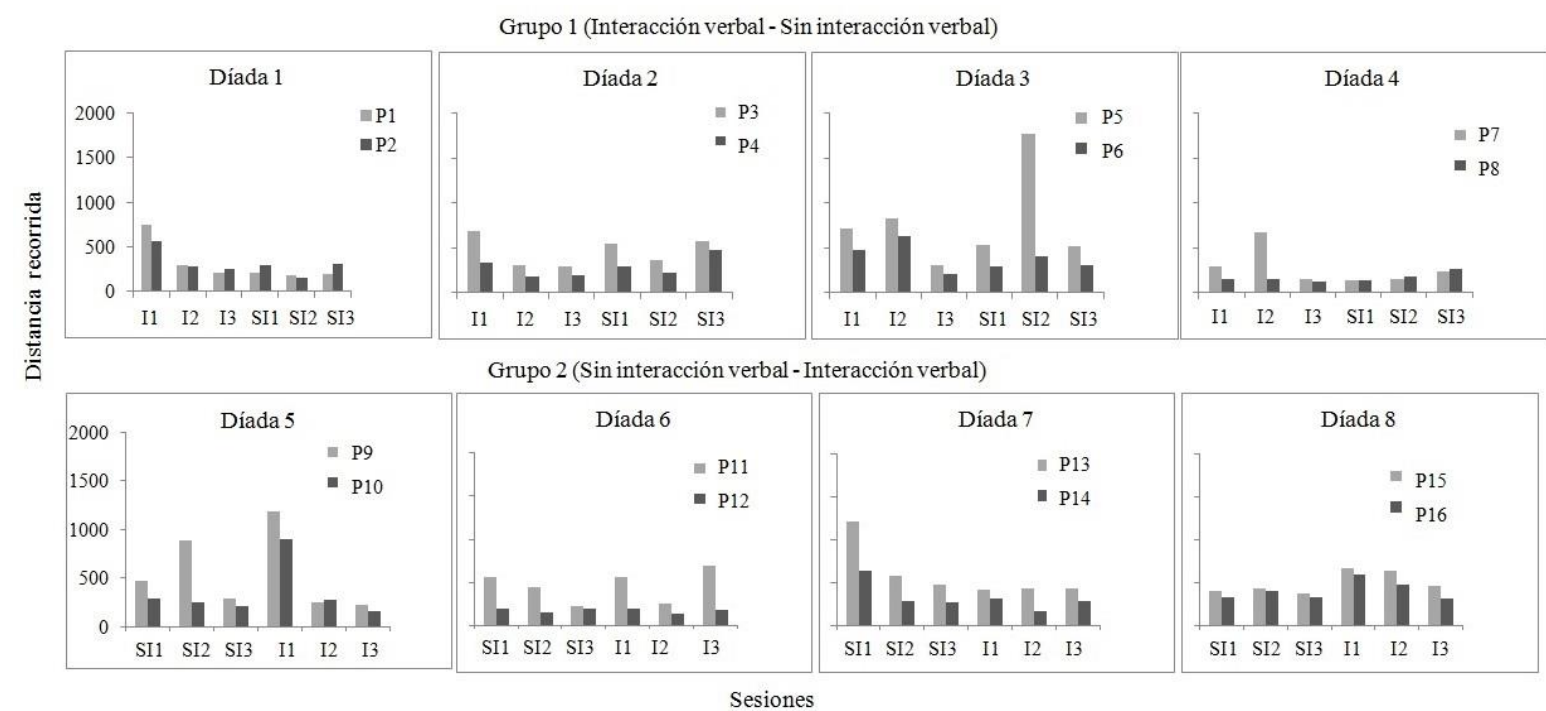

Figura 6. Distancia recorrida con los objetos en cada participante de ambos grupos. $\mathrm{P}=$ Participantes; SI = Sesiones sin interacción verbal (el número corresponde al número de sesión); I = Sesiones con interacción verbal (el número corresponde al número de sesión).

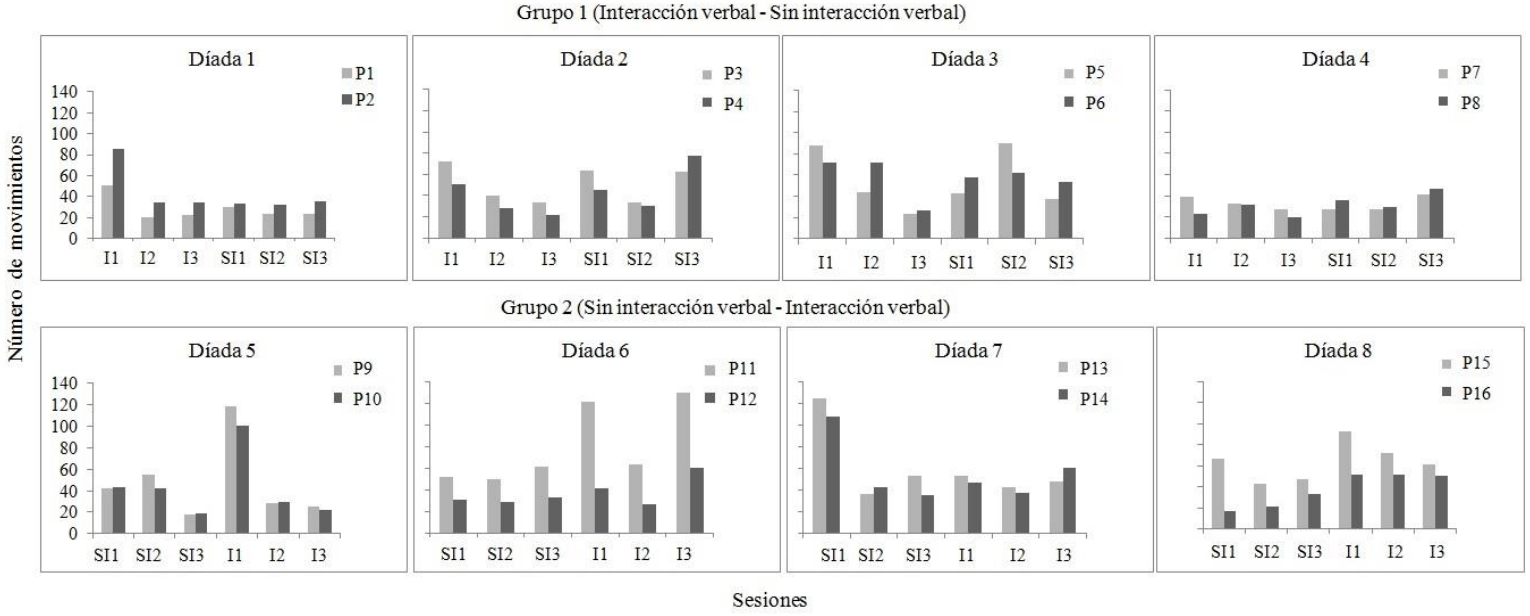

Figura 7. Número de movimientos realizados a lo largo de las sesiones por cada participante de ambos grupos. $\mathrm{P}=$ Participantes; $\mathrm{SI}=$ Sesiones sin interacción verbal (el número corresponde al número de sesión); I = Sesiones con interacción verbal (el número corresponde al número de sesión). 


\section{Discusión}

El presente trabajo tuvo como objetivo principal evaluar, en situaciones cooperativas, el efecto de permitir o restringir la interacción verbal entre los participantes sobre la ejecución de tareas que requirieron respuestas individuales. Debido a que en la literatura se ha reportado que las interacciones verbales entre los participantes han facilitado la emergencia de líderes en la situación experimental, dirigiendo y facilitando la resolución de la tarea (i.e., la eficiencia) (Bicchieri \& LevOn, 2007; Bruttel \& Fischbacher, 2013; Sutter \& Rivas, 2014) se realizó también un análisis de los comentarios directivos y/o estratégicos (de acuerdo con Pulido et al., 2013) realizados por los participantes con el objetivo de evaluar si afectaban o no la forma de realización de la tarea.

En relación con el primer objetivo, en la literatura se ha reportado ampliamente el efecto positivo que la interacción verbal tiene en la elección de respuestas cooperativas sobre las individuales (e.g., Bicchieri \& Lev-On, 2007; Cohen et al., 2010; Hake \& Schmid, 1981; Marwell \& Schmitt, 1975; Meleady et al., 2013; Pulido-Avalos et al., 2014; Ribes Iñesta et al., 2005; Wichman, 1970) y al parecer, este tipo de interacción también tiene un efecto positivo sobre el desempeño en este tipo de tareas, cuando se presentan sin la alternativa no cooperativa (Pulido et al., 2013).

De acuerdo con el efecto en el desempeño, en el presente experimento se observó que, en general, las díadas mostraron mayores índices de ineficiencia (IIneF e IIneT) cuando se expusieron a la condición de restricción verbal. Aunque dos díadas del Grupo 1 mostraron sus más altos IIneF e IIneT durante la condición en la que se permitió la comunicación entre los participantes, dichos índices fueron inferiores (por mitad) a los mostrados por las díadas que fueron ineficientes en condiciones de restricción verbal.

A pesar de la diferencia entre los procedimientos utilizados en el estudio de $\mathrm{Pu}$ lido et al. (2013), en el que se requirió de la acción de los dos participantes para realizar una respuesta, y los procedimientos del presente estudio, en el que las respuestas de los participantes fueron individuales, el efecto fue el mismo: los participantes fueron más eficientes bajo condiciones en las que se permitió el intercambio verbal entre ellos.

Por otro lado, en los procedimientos de Pulido et al. (2013) los participantes fueron expuestos solo a condiciones de restricción verbal o a condiciones en las que se permitió la comunicación entre ellos, en cambio, en el presente estudio todos los participantes fueron expuestos a las dos condiciones en diferente orden, quizá por ello, el efecto comunicación fue más claro en el primer estudio. Sin embargo, es importante mencionar que no se observó un efecto del orden de presentación de las condiciones de permisión/restricción verbal. Este hallazgo tiene sentido a la luz de la propuesta de Ribes-Iñesta (2001) y Ribes-Iñesta, Rangel y López-Valadéz (2008), debido al papel fundamental del lenguaje en las interacciones sociales. Es decir, al permitir la comunicación entre los participantes, se observó un decremento en los índices de ineficiencia, independientemente del orden en el que se presentaron las condiciones experimentales a los participantes.

Por otra parte, el que la mitad de las díadas (las díadas del Grupo 1) haya alcanzado una mayor duración en la resolución de la tarea durante SI1 (sesión con posibilidad de comunicación), pudo deberse a que esta fue la primera sesión del experi- 
mento y, probablemente por ello, a un proceso de aprendizaje sobre el funcionamiento de la tarea. Este efecto, aunque no fue tan marcado en el Grupo 2 (que inició con restricción verbal), también se presentó.

Un aspecto interesante es que en el Grupo 2 hubo un incremento de la duración en la realización de la tarea en todas las díadas durante la sesión I1 en la Fase 2, es decir, la primera en la que se permitió la comunicación entre los participantes. Sin embargo, como se mencionó anteriormente, no fue en estas sesiones de comunicación en las que las díadas mostraron mayores IIneF e InneT, por lo que, al parecer, la duración no está relacionada con los índices de ineficiencia calculados.

Esta evidencia parece ir en contra de los resultados reportados por Pulido et al. (2013), quienes observaron que los participantes que se comunicaron entre ellos fueron más eficientes en la resolución de la tarea, tanto en precisión como en el tiempo empleado para realizarla. Quizá estas diferencias puedan deberse a que las tareas utilizadas fueron distintas y requirieron de procedimientos de respuesta diferentes (compartidas, en el caso de Pulido et al. 2013, e individuales en el caso del presente trabajo).

En virtud de lo anterior, quizá sería importante evaluar, bajo la tarea experimental utilizada en este estudio, la posibilidad de resolver tareas cooperativas que requieran respuestas compartidas, tal y como lo hicieron Pulido et al. (2013); aspecto que además de permitir una comparación más cercana de los efectos del tipo de respuesta (por utilizar la misma tarea con los distintos tipos de respuesta), permitiría, a su vez, extender los hallazgos encontrados por los autores del estu- dio antecedente, al utilizar procedimientos experimentales distintos.

Respecto del objetivo de la evaluación de los comentarios directivos en la situación experimental, en general, en dos díadas del Grupo 1 (Díadas 3 y 4) y en tres díadas del Grupo 2 (6, 7 y 8), se puede observar que uno de los participantes $(6,7$, 11,14 y 16) tuvo una mayor cantidad de intervenciones directivas (ID), en relación con su compañero de díada.

Analizando este tipo de intervenciones con la ejecución individual en el desarrollo de la tarea, se puede observar que (con excepción de los participantes 7 y 11) aquellos que realizaron un mayor número de ID recorrieron menores distancias con los objetos que sus compañeros (ver figura 6). Sin embargo, al parecer, la presencia de un mayor número de ID realizadas por los participantes no tuvo efecto en el número de movimientos realizados por cada uno de ellos (ver figura 7).

Quizá estos datos se encuentren relacionados con lo que Bicchieri y Lev-On (2007) sugirieron: en situaciones en las que las interacciones son impersonales, o no se permite la comunicación entre los participantes, es probable que se obstaculice la emergencia de un líder. De acuerdo con lo anterior, probablemente las ID tengan mayores efectos sobre la ejecución en interacciones frente a frente; sin embargo, esto dificultaría la posibilidad de analizar los efectos de la ejecución en tareas cooperativas sin interacciones verbales.

Es importante señalar que, en este análisis de dirección de unos participantes sobre otros, no se está dejando de lado la estructura funcional de la interacción. Es decir, de acuerdo con lo propuesto por Rangel y Ribes (2009) y por Ribes-Iñesta, Rangel y 
López-Valadéz (2008), además del seguimiento que se presenta entre los participantes, es indispensable tener en cuenta que la interacción se enmarca dentro de un contexto particular, así como de las instituciones que la regulan. En esta situación específica, por ejemplo, ninguno de los individuos se encontraba bajo una situación de autoridad institucional respecto del otro y podría decirse que su ejecución no trascendió los límites del experimento.

Finalmente, el hecho de que a) el desempeño de una tarea que requería de la cooperación de los participantes haya mejorado una vez que se establecieron interacciones verbales entre ellos (en comparación a cuando la comunicación no fue permitida) y; b) que los participantes realizaran comentarios dirigidos a guiar la ejecución de la tarea; apoya lo que se ha afirmado por Ribes-Iñesta (2001) y Ribes-Iñesta, Rangel y LópezValadéz (2008), sobre el papel que juega el lenguaje en la posibilitación del comportamiento social.

Con referencia al papel facilitador que las interacciones verbales parecen tener en el comportamiento cooperativo, a diferencia del estudio realizado por Pulido et al. (2013), el criterio de las respuestas requeridas en este trabajo fue individual, pero al parecer, trabajar por la consecución de una meta común y, por consiguiente, estar involucrados en una contingencia compartida, parece requerir de la intervención del lenguaje para su óptimo desarrollo.

En este punto es importante tomar en cuenta que, tal como se aprecia en los datos, la tarea cooperativa puede cumplirse aún en ausencia de interacciones verbales en la situación experimental; sin embargo, la ejecución parece ser más eficiente cuando participa el lenguaje como medio para lograrlo.

Quizá estos datos puedan ser analizados en los términos propuestos por RibesIñesta, Rangel, Ramírez et al. (2008), en los que se distingue el control situacional del control lingüístico del comportamiento social y que, de acuerdo con los datos reportados por ellos, y los encontrados en este estudio, el último parece ser más efectivo cuando de comportamiento social se trata. Tal y como afirman Ribes-Iñesta (2001) y Ribes-Iñesta, Rangel y LópezValadéz (2008), lo que posibilita el comportamiento social "es un medio de contacto articulado en la forma de lenguaje y mediado por él"' (p. 45).

Con respecto a los comentarios directivos realizados por los participantes durante la ejecución de la tarea, es importante señalar que se debe tomar con precaución la medida de liderazgo sugerida en este estudio y los mencionados anteriormente en este escrito. Como se dijo, las interacciones directivas realizadas por alguno de los participantes solo fueron registradas cuando fueron atendidas por el otro. Sin embargo, aunque, tal y como señalan Rangel y Ribes (2009) y Ribes-Iñesta, Rangel y López-Valadéz (2008), este criterio es necesario, pero no es suficiente para que se pueda hablar de la existencia de una relación de liderazgo. Es decir, se sugiere que, en la lectura de esta medida, siempre se considere el contexto institucional en el que la interacción tiene lugar y el reconocimiento de la autoridad moral que identifica este tipo de relación social.

En este sentido, sería interesante explorar el efecto de este tipo de interacciones (interacciones directivas) cuando exista una autoridad formal entre los participantes o cuando estos supongan que la ejecución de 
la tarea experimental tendrá repercusiones a largo plazo.

Por otro lado, a partir de este trabajo, se ha identificado la necesidad de corroborar, procedimentalmente en siguientes estudios, la autoridad moral que Rangel y $\mathrm{Ri}$ bes (2009) y Ribes-Iñesta, Rangel y López-Valadéz (2008) afirman que se debe establecer en una relación de liderazgo entre los individuos de una interacción.

Finalmente, en el reconocimiento de que la interacción verbal no es la única forma en la que el lenguaje puede posibilitar una interacción, los resultados encontrados sugieren la relevancia de explorar y comparar los efectos de distintos tipos de interacción lingüística (e.g., oral versus escrita) en la resolución de una tarea cooperativa; así como su efecto, tanto en la ejecución como en las relaciones, entre los participantes. Lo anterior, bajo la suposición de que en el medio de contacto convencional, que se transmite por y como lenguaje, como sistemas de relaciones de convivencia (Ribes-Iñesta, Rangel, \& López-Valadéz, 2008), la forma en la que se presente esta posibilitación de interacciones podría ser menos importante que su funcionalidad cuando de estudiar el comportamiento social se trate.

\section{Referencias}

Acuña, L. (2010). El uso de la estadística en análisis de la conducta: ¿cuándo usarla y cuándo no? Revista Mexicana de Análisis de la Conducta, 36(1), 133-145. Recuperado de http://www.journals.unam.mx/index.php/rmac/article/viewFile/18020/17161

Azrin, N. H. \& Lindsley, O. R. (1956). The reinforcement of cooperation between children. The Journal of Abnormal and Social Psychology, 52(1), 100-102. http://dx.doi.org/10.1037/h0042490

Balliet, D., Mulder, L. B., \& Van Lange, P. A. M. (2011). Reward, punishment, and cooperation. A meta-analysis. Psychological Bulletin, 137(4), 594-615. http://dx.doi.org/10.1037/a0023489

Bicchieri, C. \& Lev-On, A. (2007). Computer-mediated communication and cooperation in social dilemmas: An experimental analysis. Politics, Philosophy \& Economics, 6(2), 139-168.

http://dx.doi.org/10.1177/1470594X07077267

Bruttel, L. \& Fischbacher, U. (2013). Taking the initiative. What characterizes leaders? European Economic Review, 64, 147-168. http://dx.doi.org/10.1016/j.euroecorev.2013.08.008

Cohen, T. R., Wildschut, T., \& Insko, C. A. (2010). How communication increases interpersonal cooperation in mixed-motive situations. Journal of Experimental Social Psychology, 46(1), 39-50.

http://dx.doi.org/10.1016/j.jesp.2009.09.009

Dawes, R. M. \& Messick, D. M. (2000). Social dilemmas. International Journal of Psychology, 35(2), 111-116. http://dx.doi.org/10.1080/002075900399402 
Etkin, W. (1964). Social behavior from fish to man. London, England: The Chicago University Press.

Hake, D. F., Olvera, D., \& Bell, J. C. (1975). Switching from competition to sharing or cooperation at large response requirements: Competition requires more responding. Journal of the Experimental Analysis of Behavior, 24(3), 343-354.

http://dx.doi.org/10.1901/jeab.1975.24-343

Hake, D. F. \& Schmid, T. L. (1981). Acquisition and maintenance of trusting behavior. Journal of the Experimental Analysis of Behavior, 35(1), 109-124.

http://dx.doi.org/10.1901/jeab.1981.35-109

Hake, D. F. \& Vukelich, R. (1972). A classification and review of cooperation procedures. Journal of the Experimental Analysis of Behavior, 18(2), 333-343. http://dx.doi.org/10.1901/jeab.1972.18-333

Marwell, G. \& Schmitt, D. R. (1975). Cooperation: An experimental analysis. New York, New York: Academic Press.

Matthews, B. A. (1977). Magnitudes of score differences produced within sessions in a cooperative exchange procedure. Journal of the Experimental Analysis of Behavior, 27(2), 331-340. http://dx.doi.org/10.1901/jeab.1977.27-331

Meleady, R., Hopthrow, T., \& Crisp, R. J. (2013). Simulating social dilemmas: Promoting cooperative behavior through imagined group discussion. Journal of Personality and Social Psychology, 104(5), 839-853. http://dx.doi.org/10.1037/a0031233

Pierce, W. D. (1991). Culture and society: The role of behavioral analysis. En P. A. Lamal (Ed.), Behavioral analysis of societies and cultural practices (pp. 13-37). New York, New York: Hemisphere Publishing Corporation.

Pulido Avalos, L. (2012). Efecto del tipo de contingencia en el establecimiento y cualidad de intercambios verbales: su papel en la elección de alternativas compartidas (Tesis doctoral inédita). Universidad de Guadalajara, Guadalajara, México.

Pulido Avalos, L., Rangel Bernal, N., Ávila Hernández, A. K., Mérida Velez, F. Y., \& Ribes Iñesta, E. (2014). Efecto del intercambio de recursos y fuerza de trabajo en la elección de contingencias compartidas. Acta Comportamentalia, 22(3), 295-314. Recuperado de https://is.gd/xEzTnm

Pulido, L., Rangel, N. E., \& Ortiz, G. (2013). El papel del intercambio verbal en la solución de tareas en niños de primaria. Acta Comportamentalia, 21(1), 36-52. Recuperado de http://www.revistas.unam.mx/index.php/acom/article/view/36628

Pulido-Avalos, L., Rangel-Bernal, N., \& Ortiz-Rueda, G. (2014). Efecto del tipo de contingencia en el establecimiento y cualidad de intercambios verbales: su papel en la elección de alternativas compartidas. Journal of Behavior, Health \& Social Issues, 6(1), 71-86. Recuperado de http://www.sciencedirect.com/science/article/pii/S20070780153000671 
Rangel, N. \& Ribes, E. (2009). Un análisis experimental del poder y la autoridad. En M. A. Padilla Vargas (Ed.), Avances en la investigación del comportamiento animal y humano, (pp. 141-153). Guadalajara, México: Universidad de Guadalajara.

Ribes-Iñesta, E. (2001). Functional dimensions of social behavior: Theoretical considerations and some preliminary data. Revista Mexicana de Análisis de la Conducta, 27(2), 285-306. Recuperado de https://is.gd/dUyz8S

Ribes-Iñesta, E., Rangel, N. E., \& López-Valadéz, F. (2008). Análisis teórico de las dimensiones funcionales del comportamiento social. Revista Mexicana de Psicología, 25(1), 45-57. Recuperado de http://www.redalyc.org/articulo.oa?id=243016300003

Ribes Iñesta, E., Rangel, N., Magaña, C., López, A. G., \& Zaragoza, A. (2005). Efecto del intercambio diferencial equitativo e inequitativo en la elección de contingencias sociales de altruismo parcial. Acta Comportamentalia, 13(2), 159-179. Recuperado de http://www.revistas.unam.mx/index.php/acom/article/view/14546

Ribes-Iñesta, E., Rangel, N., Pulido, L., Valdez, U., Ramírez, E., Jiménez, C., \& Hernández, M. (2010). Reciprocity of responding as a determinant of partial-altruistic behavior in humans. European Journal of Behavior Analysis, 11(2), 105-114. http://dx.doi.org/10.1080/15021149.2010.11434337

Ribes-Iñesta, E., Rangel, N., Ramírez, E. Valdez, U., Romero, C., \& Jiménez, C. (2008). Verbal and-nonverbal induction of reciprocity in a partial-altruism social interaction. European Journal of Behavior Analysis, 9(1), 53-72.

http://dx.doi.org/10.1080/15021149.2008.11434295

Ribes-Iñesta, E., Rangel, N., Zaragoza, A., Magaña, C., Hernández, H., Ramírez, E. \& Valdez, U. (2006). Effects of differential and shared consequences on choice between individual and social contingencies. European Journal of Behavior Analysis, 7(1), 41-56. http://dx.doi.org/10.1080/15021149.2006.11434262

Sally, D. (1995). Conversation and cooperation in social dilemmas. Racionality and Society, 7(1), 58-92. http://dx.doi.org/10.1177/1043463195007001004

Schmid, T. L. \& Hake, D. F. (1983). Fast acquisition of cooperation and trust: A twostage view of trusting behavior. Journal of the Experimental Analysis of Behavior, 40(2), 179-192.

http://dx.doi.org/10.1901/jeab.1983.40-179

Schmitt, D. R. (1984). Interpersonal relations: Cooperation and competition. Journal of the Experimental Analysis of Behavior, 42(3), 377-383.

http://dx.doi.org/10.1901/jeab.1984.42-377

Schmitt, D. R. (1986). Competition: Some behavioral issues. The Behavior Analyst, 9(1), 2734. Recuperado de http://www.ncbi.nlm.nih.gov/pmc/articles/PMC2741880/ 
Schmitt, D. R. (1987). Interpersonal contingencies: Performance differences and costeffectiveness. Journal of the Experimental Analysis of Behavior, 48(2), 221-234. http://dx.doi.org/10.1901/jeab.1987.48-221

Schmitt, D. R. (1998). Social behavior. En K. A. Lattal \& M. Perone (Eds.), Handbook of research methods in human operant behavior (pp. 471-505). New York, New York: Springer US. http://dx.doi.org/10.1007/978-1-4899-1947-2_15

Schmitt, D. R. (2000). Effects of competitive reward distribution on auditing and competitive responding. Journal of the Experimental Analysis of Behavior, 74(1), 115-125.

http://dx.doi.org/10.1901/jeab.2000.74-115

Secord, P. F. \& Backman, C. W. (1974). Social psychology. New York, New York: McGraw Hill.

Shimoff, E. \& Matthews, B. A. (1975). Unequal reinforcer magnitudes and relative preference for cooperation in the dyad. Journal of the Experimental Analysis of Behavior, 24(1), 1-16. http://dx.doi.org/10.1901\%2Fjeab.1975.24-1

Skinner, B. F. (1953). Science and human behavior. New York, New York: The Free Press.

Spiga, R., Cherek, D. R., Grabowski, R., \& Bennet, R. H. (1992). Effects of inequity on human free operant cooperative responding: A validation study. The Psychological Record, 42(1), 29-40. Recuperado de http://psycnet.apa.org/psycinfo/1992-26197-001

Sutter, M. \& Rivas, M. F. (2014). Leadership, reward and punishment in sequential public goods experiments. En P. A. M. Van Lange, B. Rockenbach, \& T. Yamagishi (Eds.), Reward and punishment in social dilemmas (pp. 133-155). New York, New York: Oxford University Press.

http://dx.doi.org/10.1093/acprof:oso/9780199300730.003.0008

Uscanga, D. (2011). aTube Catcher (Versión 3.8). [Software computacional]. Recuperado de http://www.atube.me/video/index.html

Wichman, H. (1970). Effects of isolation and communication on cooperation in a twoperson game. Journal of Personality and Social Psychology, 16(1), 114-120.

http://dx.doi.org/10.1037/h0029845 